\title{
Lipoabdominoplastia ampliada associada a múltiplos procedimentos cirúrgicos
}

\author{
Amplified lipoabdominoplasty associated with multiple surgical procedures
}

Claudia Francisco

Oliveira $^{1}$

Trabalho realizado no Hospital Antoninho da Rocha Marmo e Clínica Vanité, São José dos Campos, SP, Brasil.

Artigo submetido pelo SGP (Sistema de Gestão de Publicações) da RBCP.

Artigo recebido: 20/8/2010 Artigo aceito: 22/2/2011

\begin{abstract}
RESUMO
Introdução: A lipoabdominoplastia tem se mostrado uma técnica segura, com obtenção de bons resultados estéticos, trazendo uma outra visão à abordagem do contorno corporal. Objetivos: Este trabalho demonstra uma experiência pessoal, com a lipoabdominoplastia, descolando de forma ampla as regiões periumbilical e supraumbilical, com base na anatomia vascular da parede abdominal em sua porção lateral. O trabalho também demonstra a associação frequente a múltiplos procedimentos cirúrgicos. Método: Foram tratados 138 pacientes, no período de janeiro de 2002 a dezembro de 2009, que tinham indicação tanto para abdominoplastia clássica quanto para a lipoaspiração de abdome simultaneamente. Foram excluídos pacientes com obesidade mórbida e aqueles sem condições clínicas para cirurgia. A cirurgia foi realizada fazendo-se a lipoabdominoplastia com o descolamento do retalho supraumbilical e da região periumbilical, mais ampliado lateralmente, sendo que o retalho infraumbilical não foi lipoaspirado, sendo removido em bloco com a fáscia de Scarpa. Em associação à lipoabdominoplastia foi realizada a lipoaspiração de, no mínimo, três regiões (dorso, flancos e abdome), muita vezes, associada a outros procedimentos de cirurgia plástica ou cirurgias de outras especialidades $(50 \%$ dos pacientes). Resultados: Todos os procedimentos tiveram resultados considerados satisfatórios pelos pacientes, realizados num tempo médio de 3 horas, com complicações locais pequenas, mas de fácil resolução. Conclusões: A lipoabdominoplastia sem lipoaspiração do retalho infraumbilical e com remoção da fáscia de Scarpa, mesmo sendo feita com um descolamento maior, nas regiões mediana periumbilical e supraumbilical, demonstrou ser uma cirurgia segura, podendo ser associada a outros procedimentos cirúrgicos.
\end{abstract}

Descritores: Abdome/cirurgia. Lipectomia. Cirurgia plástica/métodos.

\begin{abstract}
Introduction: Lipoabdominoplasty has been as a safe showed technique with good aesthetic results, bringing a new view to the approach to the body's contour. Objectives: This study shows a personal experience with lipoabdominoplasty, widely detaching the periumbilical and supraumbilical areas, based on the vascular anatomy of the abdominal wall in its lateral section. The study also shows the frequent association with multiple surgical procedures. Methods: 138 patients were treated during the period from January 2002 to December 2009, who had a recommendation for both classical abdominoplasty and simultaneous liposuction of the abdomen. Patients with morbid obesity or with no clinical conditions for surgery were excluded. Surgery was carried out by doing the lipoabdominoplasty with the detachment of supraumbilical cutting and of the perimbilical area, more amplified laterally, whereas the infraumbilical cutting was not submitted to liposuction, but removed in its entirety together with Scarpa's fascia. In conjunction with the lipoabdominoplasty, liposuction was done in at least three areas (back, flanks and abdomen), often associated with other plastic surgery procedures or surgeries of other specialties (total of fifty percent of patients). Results: All the procedures had outcomes deemed satisfactory by patients, carried out on the average of three hours, with minor local complications that were easily solved. Conclusion: Lipoabdominoplasty without liposuction of the infraumbilical cutting and with the removal of Scarpa's fascia, even though carried out with a larger detachment in the periumbilical and supraumbilical areas, proved to be a secure surgery, capable of being associated with other surgical procedures.
\end{abstract}

Keywords: Abdome/surgery. Lipectomy. Plastic surgery/methods.

1. Membro Titular da Sociedade Brasileira Cirurgia Plástica; Diretora e Proprietária da Clínica Vanité, São José dos Campos, SP, Brasil. 


\section{INTRODUÇÃO}

Técnicas de abdominoplastias descritas por Pitanguy ${ }^{1} \mathrm{e}$ Callia ${ }^{2}$ iniciaram uma busca por melhores resultados estéticos e estimularam outros autores a descreverem novas variantes.

Com o advento da lipoaspiração, descrita por Illouz ${ }^{3}$, em 1980, o tratamento da região abdominal ganhou especial atenção, no sentido de melhorar o resultado não só por meio da remoção cutânea, mas com a redução do conteúdo de gordura no contorno corporal. Em 1992, Illouz descreveu uma técnica de abdominoplastia sem descolamento do retalho supraumbilical, com lipoplastia desta área e neoumbilicoplastia, somente em pacientes com abdome em avental.

O estudo vascular dos retalhos miocutâneos realizado por Mathes e Nahai ${ }^{4}$ classifica o músculo reto abdominal como tipo II, tendo dois pedículos vasculares principais que geram as artérias perfurantes, nutridoras da porção mediana da parede abdominal, principalmente na região periumbilical.

Segundo Huger ${ }^{5}$, a anatomia vascular da parede abdominal engloba três zonas:

- zona mediana - do apêndice xifóide ao púbis, entre os rebordos laterais dos músculos reto abdominais, suprida pelas artérias epigástricas inferiores e superiores profundas, com suas perfurantes;

- zona trapezoidal - entre a crista ilíaca ântero-superior superiormente e a região inguinal inferiormente, com aporte vascular vindo das circunflexas ilíacas superficiais, epigástricas inferiores superficiais e artérias pudendas externas (sistema superficial) e epigástricas inferiores profundas (sistema profundo);

- área do abdome lateral e flancos - suprida pelas artérias subcostais, lombares segmentares e intercostais.

A abdominoplastia tradicional, com sua incisão transversa baixa e amplo descolamento até o rebordo costal, sacrifica as zonas 1 e 2 e parte da zona 3. Além disso, a pele da zona 1 sofre comprometimento vascular adicional pela tensão na linha de sutura e afinamento do retalho.

Muitos cirurgiões propõem um procedimento menor, com o intuito de manter a circulação do retalho preservada. Para evitar o comprometimento vascular, a lipoaspiração persiste como um procedimento independente da abdominoplastia, a ser realizada após 6 meses.

Em 1985, Hakme $^{6}$ descreveu a miniabdominoplastia, remoção de uma faixa de pele suprapúbica associada à lipoaspiração. Shestak ${ }^{7}$, em 1999, relatou remoção parcial de pele de região suprapúbica sem transposição de cicatriz umbilical.

Em 2000, Avelar ${ }^{8}$ reportou nova técnica de abdominoplastia sem descolamento, com remoção de gordura, a lipoaspiração de região submamária com miniabdominoplastia.

No ano seguinte, Saldanha et al. ${ }^{9}$ descreveram técnica que associa lipoaspiração do abdome sem descolamento do retalho supraumbilical, com preservação da fáscia de
Scarpa com uma fina camada de gordura de todo o retalho infraumbilical lipoaspirado, seguida de onfaloplastia. Em 2003, Saldanha et al. ${ }^{10}$, novamente, descreveram a lipoabdominoplastia, mas com descolamento supraumbilical seletivo e seguro, preservando ainda uma fina camada de gordura sob a fáscia de Scarpa na porção infraumbilical.

Graf et al. ${ }^{11}$, em 2006, utilizaram Dopplerfluxometria para avaliar os vasos perfurantes periumbilicais nos períodos pré e pós-operatório, a fim de fazer um descolamento reduzido associado à abdominoplastia clássica.

Uebel $^{12}$, em 2008, descreveu a lipoabdominoplastia com remoção do retalho abdominal infraumbilical em bloco, com preservação de uma faixa da fáscia de Scarpa e ressecção do retalho suprapúbico por incisão superficial ao tecido subcutâneo. Também em 2008, Vila-Rovira ${ }^{13}$ relatou técnica de extensa lipoaspiração com mínima abdominoplastia.

\section{MÉTODO}

No período de janeiro de 2002 a dezembro de 2009, 138 pacientes com indicação tanto para abdominoplastia clássica quanto para a lipoaspiração de abdome simultaneamente foram operados. Os pacientes faziam parte da clínica privada da autora e todos os procedimentos foram realizados em ambiente hospitalar, o Hospital Antoninho da Rocha Marmo, em São José dos Campos, SP, Brasil.

Todos os pacientes foram submetidos a avaliação préoperatória, incluindo exames laboratoriais de sangue, eletrocardiograma e consulta pré-anestésica.

Foram incluídas pacientes diabéticas e hipertensas compensadas ou com sobrepeso. Pacientes tabagistas foram incluídas no estudo desde que suspendessem o cigarro 15 dias antes e após a operação. Também foi solicitada a suspensão do anticoncepcional oral pelo mesmo período.

Todos os pacientes apresentavam flacidez cutânea supra e infraumbilical, suficiente para indicar abdominoplastia e lipodistrofia de dorso, laterais e abdome, que indicassem a necessidade de lipoaspiração.

Os procedimentos foram realizados em regime hospitalar, com internação de 24 horas. Foram realizados um ou dois bloqueios do tipo peridural com sedação, feitos de acordo com a localização das cirurgias associadas: região infraescapular, mama ou membros inferiores.

Compressão intermitente de panturrilhas foi utilizada a partir do ato cirúrgico e mantida até a deambulação, normalmente no dia seguinte à operação. Heparina de baixo peso molecular foi empregada somente em alguns casos específicos, como portadores de diabetes, idosos e tabagistas. Quando usada, heparina foi suspensa no dia seguinte ao deambular. Deambulação precoce e constante foi uma orientação permanente.

Realizada infiltração de solução de soro fisiológico com adrenalina a 1:400.000, na proporção de $1: 1$, sem técnica 
tumescente. Realizada vibrolipoaspiração com cânulas de 3 e $4 \mathrm{~mm}$, de 3 e 4 furos, com vibrolipoaspirador. A técnica da lipoaspiração prosseguiu com avaliação do resultado por meio da palpação, para sentir se realmente o retalho foi afinado.

As áreas comuns em todos os pacientes foram dorso, flancos e porção total do abdome, com exceção a infraumbilical. Em nenhum dos casos foi feita a lipoaspiração no retalho abdominal inferior.

Realizadas hidratação venosa per operatória com 10 $\mathrm{ml}$ por $\mathrm{kg}$, bem como antibioticoterapia venosa profilática (cefazolina) e terapêutica (cefalexina), mantida por sete dias.

A técnica de abdominoplastia foi a tradicional, com incisão horizontal suprapúbica e ramos laterais oblíquos ascendentes lateralmente, sendo a porção central cortada $1 \mathrm{a} 2 \mathrm{~cm}$ mais alta e ligeiramente curva. Realizado descolamento do retalho abdominal inferior da aponeurose anterior dos músculos retos abdominais e da musculatura oblíqua lateral, sem lipoaspiração e sem preservação da fáscia de Scarpa, até a cicatriz umbilical. Através de incisão triangular da cicatriz umbilical, realizada liberação desta com preservação de pequeno panículo adiposo ao redor.

$\mathrm{Na}$ região periumbilical, perfurantes são identificadas e, na sua maioria, seccionadas. Iniciado descolamento ampliado do rebordo medial dos músculos retos abdominais. A liberação dos músculos reto abdominais na região supraumbilical foi feita por uns 6 a $8 \mathrm{~cm}$, lateralmente ao rebordo medial, de cada lado, totalizando 12 a $16 \mathrm{~cm}$ de extensão no sentido horizontal, até o aparecimento de perfurantes superiores, localizadas mais lateralmente (Figura 1). Quando havia diástase dos músculos reto abdominais, estas medidas aumentavam conforme a extensão do afastamento, porém, mantendo os mesmos $6 \mathrm{~cm}$ de liberação para cada lado sobre os retos. Realizada plicatura dos músculos reto abdominais, com chuleio contínuo interrompido de fio de Prolene 0 , em todos os pacientes, independente da presença ou não de diástase.
Após lavagem com soro fisiológico, realizadas hemostasia e colocação de dreno de sucção a vácuo.

Para realizar o fechamento sem tensão nos hipocôndrios, foi feita ampliação do descolamento lateralmente, tanto na região supraumbilical quanto na região periumbilical, sendo seccionadas as perfurantes periumbilicais. Em seguida, o paciente foi colocado em posição de Fowler, realizada confirmação do local em que o retalho abdominal seria seccionado inferiormente. Quando possível, o excedente inferior de ilha cutânea, deixado na região suprapúbica por segurança, foi removido, produzindo descida maior da cicatriz horizontal, principalmente na porção central.

Toda e qualquer tensão na aproximação do retalho levou à ampliação do descolamento, lateralmente à cicatriz umbilical. Não foram passados pontos de aproximação do subcutâneo à aponeurose anterior com intenção de reduzir o seroma. Procedeu-se ao fechamento por planos, à incisão em estrela no retalho abdominal e à fixação da cicatriz umbilical. Colocada cinta compressiva.

No período de pós-operatório imediato, foi feito acompanhamento diário, nos 3 primeiros dias, na residência do paciente, realizado por uma enfermeira, e no quarto dia pósoperatório, o paciente iniciou o acompanhamento médico, em consultório, sendo visto a cada dois ou três dias até o fim do primeiro mês, com início das sessões de drenagem linfática. O acompanhamento mais intenso persistiu até o segundo mês.

O dreno a vácuo foi removido no segundo dia de pósoperatório na maior parte dos casos.

\section{RESULTADOS}

A idade média dos pacientes incluídos neste estudo foi de 44 anos, variando de 19 a 69 anos (Tabela 1 e Figura 2).

A duração do procedimento variou de 3 (lipoabdominoplastia dorso, flancos e abdome) até 6 horas (cirurgias
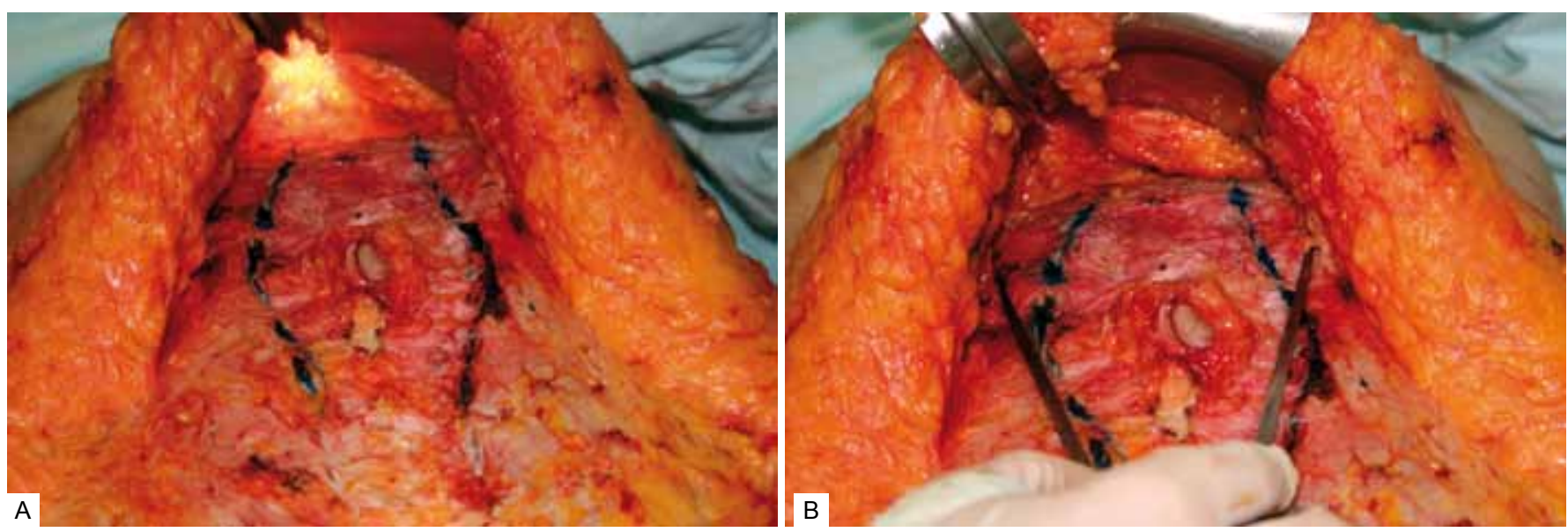

Figura 1 - Cirurgia de abdome. A: área descolada com $6 \mathrm{~cm}$ de diástase dos músculos reto abdominais; B: total de área descolada $18 \mathrm{~cm}$. 


\begin{tabular}{c|c}
\hline \multicolumn{2}{c}{ Tabela 1 - Faixa etária. } \\
\hline Faixa etária & N \\
\hline 19 anos & 1 \\
\hline 20 a 29 anos & 20 \\
\hline 30 a 39 anos & 54 \\
\hline 40 a 49 anos & 40 \\
\hline 50 a 59 anos & 19 \\
\hline 60 a 69 anos & 4 \\
\hline
\end{tabular}

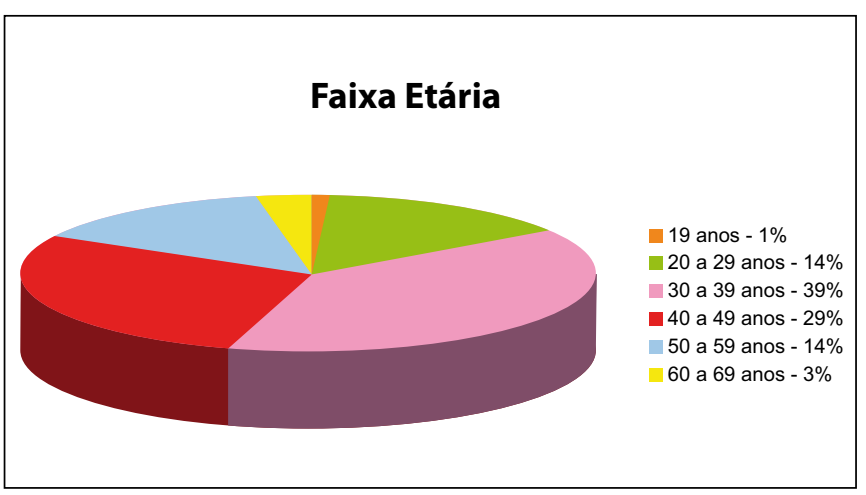

Figura 2 - Faixa etária de cirurgia plástica realizada de 2002 a 2009.

associadas de outras regiões). O volume de gordura aspirado variou de 1200 a $2000 \mathrm{ml}$.

Dentre os 138 pacientes, 35 fizeram lipoaspiração ou lipoenxertia em outras áreas além da lipoaspiração de dorso, laterais e abdome superior. Estas áreas foram infraescapulares (23), face interna de coxas (11) e culotes (8) - (Tabela 2 e Figura 3).

Sessenta e nove $(50 \%)$ pacientes realizaram outras cirurgias, além da lipoaspiração. As cirurgias plásticas associadas mais frequentes foram mamoplastia redutora (27), colocação de prótese (26) e blefaroplastia (9) - (Tabela 3 e Figura 4).

As cirurgias de outras especialidades foram histerectomias (3), colecistectomias por vídeo (3) e hernioplastias (3) - (Tabela 4 e Figura 5).

Complicações como seromas foram muito comuns, resolvendo-se logo nos primeiros dias de pós-operatório, com necessidade de punções aspirativas em 20 casos (Tabela 5 e Figura 6).

Cicatrizes inestéticas, hipertróficas ou alargadas, na região central da cicatriz horizontal, foram observadas em 15 casos. Essas pacientes foram submetidas a retoques de cicatrizes e a pequenas áreas de lipoaspiração. Abscesso localizado na porção lateral da incisão ocorreu em um caso de cirurgia combinada a mastopexia com colocação de prótese e histerectomia numa paciente obesa.

\begin{tabular}{c|c}
\hline \multicolumn{2}{c}{ Tabela 2 - Outras áreas de lipoaspiração. } \\
\hline Área & $\mathbf{N}$ \\
\hline Infraescapular & 23 \\
\hline Face interna de coxa & 11 \\
\hline Culote & 8 \\
\hline Lipoenxertia glútea & 5 \\
\hline Face interna de joelho & 4 \\
\hline Axila & 3 \\
\hline Braço & 3 \\
\hline Mento & 3 \\
\hline
\end{tabular}

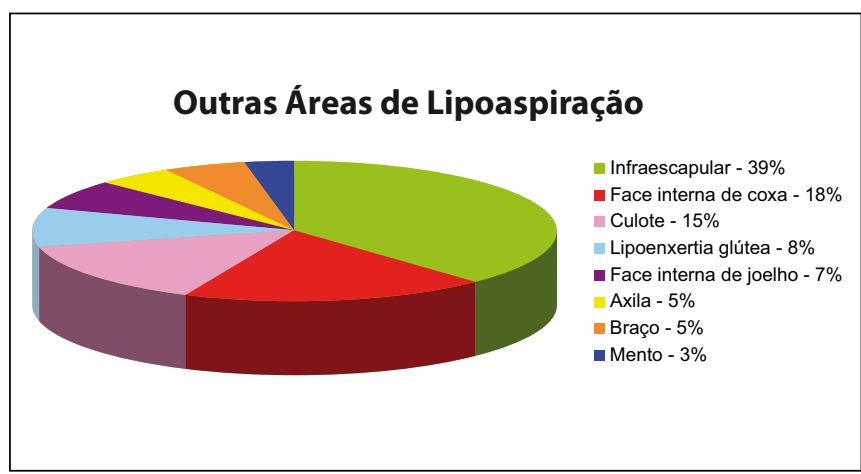

Figura 3 - Áreas de lipoaspiração associadas de cirurgia plástica realizada de 2002 a 2009.

\begin{tabular}{c|c}
\hline \multicolumn{2}{c}{ Tabela 3 - Cirurgia plástica combinada. } \\
\hline Procedimento & N \\
\hline Redução mama & 27 \\
\hline Prótese & 26 \\
\hline Blefaroplastia & 9 \\
\hline Lifting de coxa & 4 \\
\hline Laser $\mathrm{CO}_{2}$ face & 3 \\
\hline Lifting de face & 1 \\
\hline
\end{tabular}

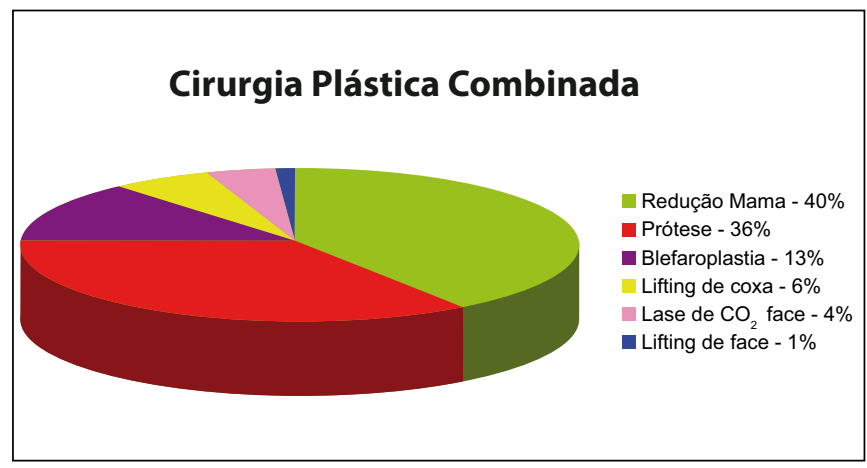

Figura 4-Cirurgias plásticas combinadas realizadas de 2002 a 2009. 


\begin{tabular}{c|c}
\hline \multicolumn{2}{c}{ Tabela 4 - Cirurgias de outras áreas. } \\
\hline Procedimento & N \\
\hline Histerectomia & 3 \\
\hline Hernioplastia & 3 \\
\hline Colecistectomia & 3 \\
\hline Cirúrgia de Varizes & 2 \\
\hline Perineoplastia & 2 \\
\hline
\end{tabular}

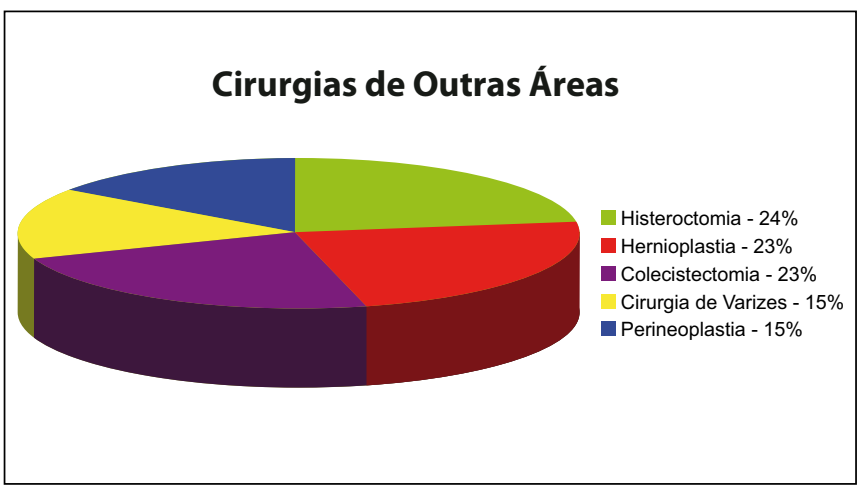

Figura 5 - Cirurgias de outras áreas realizadas de 2002 a 2009.

\begin{tabular}{c|c}
\hline \multicolumn{2}{c}{ Tabela 5 - Complicações. } \\
\hline Complicações & N \\
\hline Seromas & 20 \\
\hline Cicatrizes inestéticas & 15 \\
\hline Correções cirúrgicas & 15 \\
\hline Infecção & 1 \\
\hline
\end{tabular}

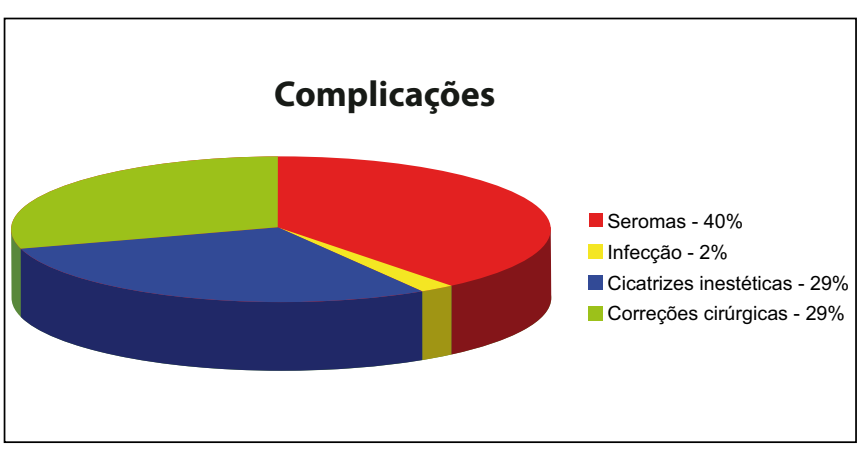

Figura 6 - Complicações de 2002 a 2009.

Nenhum caso de necrose ou epidermólise, parestesia de coxa, recidivas de diástase ou hérnias, hematomas foi evidenciado. Não houve necessidade de transfusão sanguínea.
Apesar do uso da heparina de baixo peso molecular não ter sido preconizado para todos os pacientes, foi indicado em pacientes tabagistas ou diabéticos. A administração de heparina foi feita conforme preconizado, com relação ao tempo de realização da punção peridural para o bloqueio, e sendo suspensa ao deambular, o que aconteceu logo no primeiro dia de pós-operatório.

Todas as pacientes apresentaram elevado grau de satisfação com o resultado e nenhuma se arrependeu de ter feito a cirurgia (Figuras 7 a 10).

\section{DISCUSSÃO}

Após realizar várias reconstruções mamárias com retalhos miocutâneos do abdome, que exigem enorme descolamento da parede abdominal, fica evidente que a circulação lateral da parede abdominal realmente é muito importante para a manutenção vascular do retalho abdominal ${ }^{14-16}$. Mesmo quando se faz a reconstrução das mamas bilateralmente, em que o descolamento superior e inferior é muito extenso, os dois músculos reto abdominais são transpostos com suas perfurantes periumbilicais, sendo as demais perfurantes seccionadas na zona mediana, assim como algumas subcostais e ramos das intercostais. Neste caso são seccionadas as artérias das zonas 1 e 2 e parte da 3 . Ainda assim, a parede continua suprida pelos ramos das lombares e de poucas subcostais e alguns ramos das intercostais.

Trazendo a facilidade de associar remoção de excesso cutâneo ao tratamento da lipodistrofia abdominal num único procedimento, a lipoabdominoplastia permite um resultado estético satisfatório com número de complicações locais e sistêmicas menor do que nas abdominoplastia tradicionais.

Matarasso et al. ${ }^{17}$ demonstraram, após pesquisa realizada por meio de formulários preenchidos por vários cirurgiões plásticos americanos, englobando um total de 11.000 procedimentos, que dentre as principais complicações graves em abdominoplastias estão a trombose venosa profunda e a embolia pulmonar, com incidência de $0,04 \%$ e $0,02 \%$, respectivamente. As complicações não tiveram relação como tempo de experiência dos cirurgiões.

Um estudo realizado pelo TOPS ${ }^{18}$ (Traking Operations and Outcomes for Plastic Surgeons) e o Cosmetic Assure ${ }^{19}$, nos EUA, de 2003 a 2007, demonstrou também taxas reduzidas de complicações nas abdominoplastias: hematomas $(0,9 \%)$, infecção $(3,5 \%)$, TVP e embolia pulmonar $(0,3 \%)$.

Já estudos de clínicas privadas, como os de Stewart et al. $^{20}$, de Londres, e o Neoman \& Hausen ${ }^{21}$, da Califórnia, demonstram complicações em torno de $18 \%$ e $37 \%$, respectivamente, englobando seromas (5 a 10\%), infecções ( $3 \%$ e $0)$, hematomas ( $3 \%$ e 0$)$, necroses de pele e gordura $(2,5 \%$ e 0$)$ e cicatrizes inestéticas $(23 \%$ e $7,9 \%)$. As revisões cirúrgicas foram feitas em $24 \%$ e $6,4 \%$ dos pacientes destes 

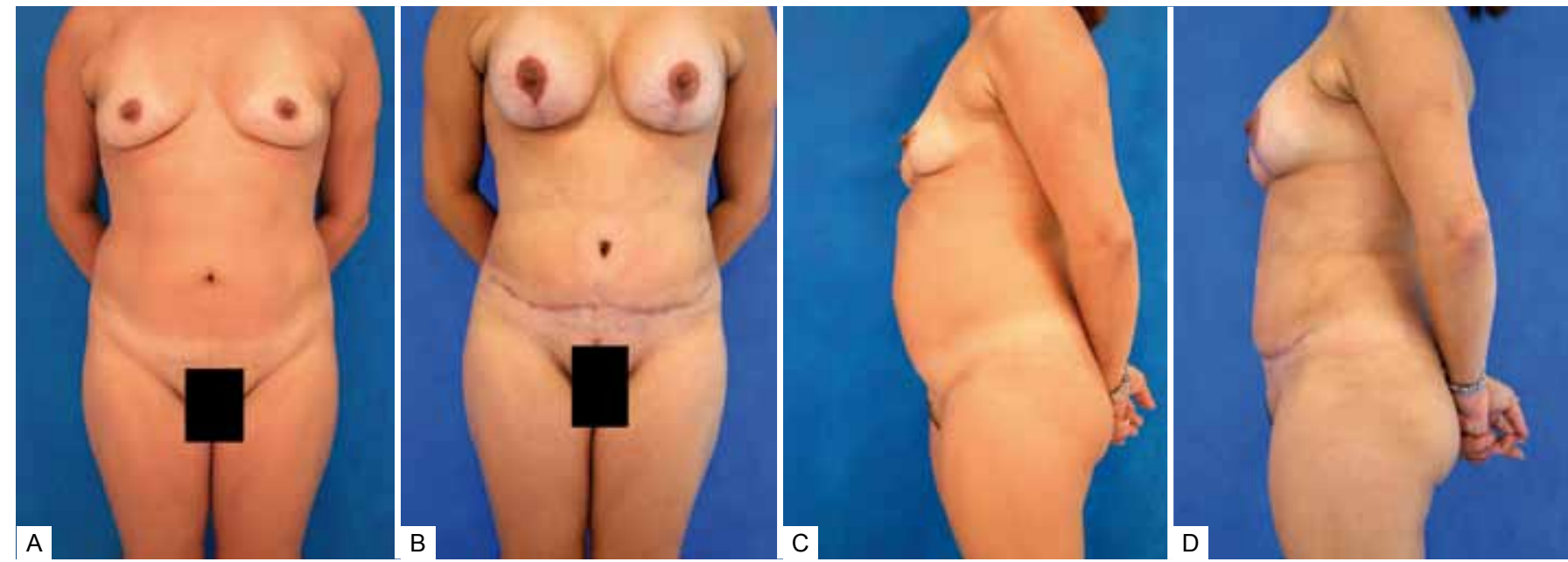

Figura 7 -Lipoabdominoplastia e mastopexia com colocação de prótese. A e $\boldsymbol{C}:$ pré-operatório; $\boldsymbol{B}$ e $\boldsymbol{D}:$ pós-operatório.
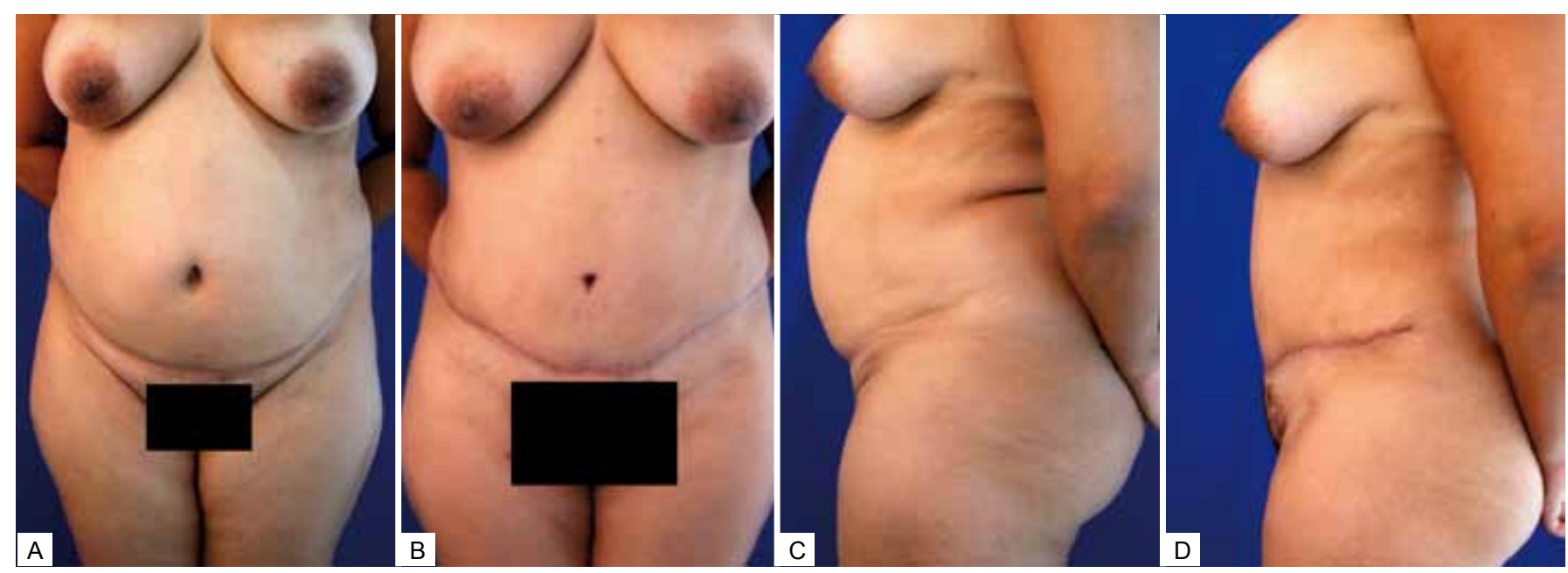

Figura 8 - Lipoabdominoplastia com lipoaspiração também de regiões infraescapular e face interna coxa. $\boldsymbol{A}$ e $\boldsymbol{C}$ : pré-operatório; $\boldsymbol{B}$ e $\boldsymbol{D}:$ pós-operatório.
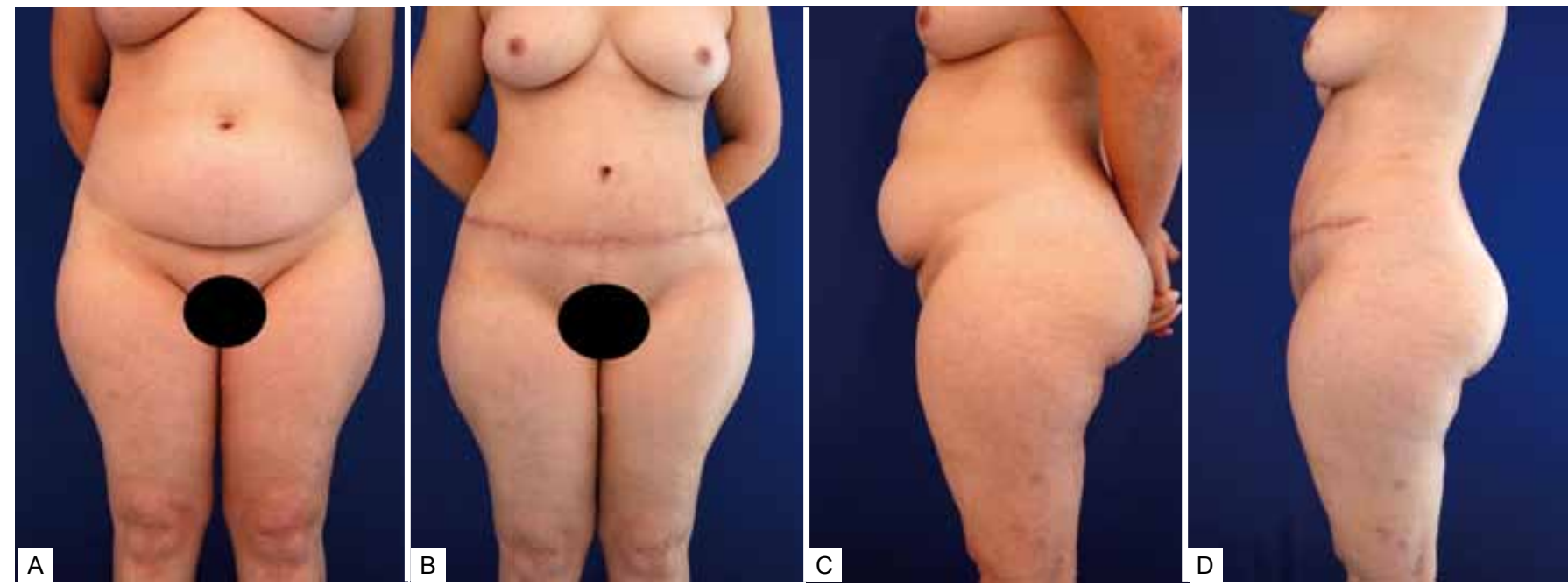

Figura 9 -Lipoabdominoplastia. A e $\boldsymbol{C}$ : pré-operatório; $\boldsymbol{B}$ e $\boldsymbol{D}:$ pós-operatório. 

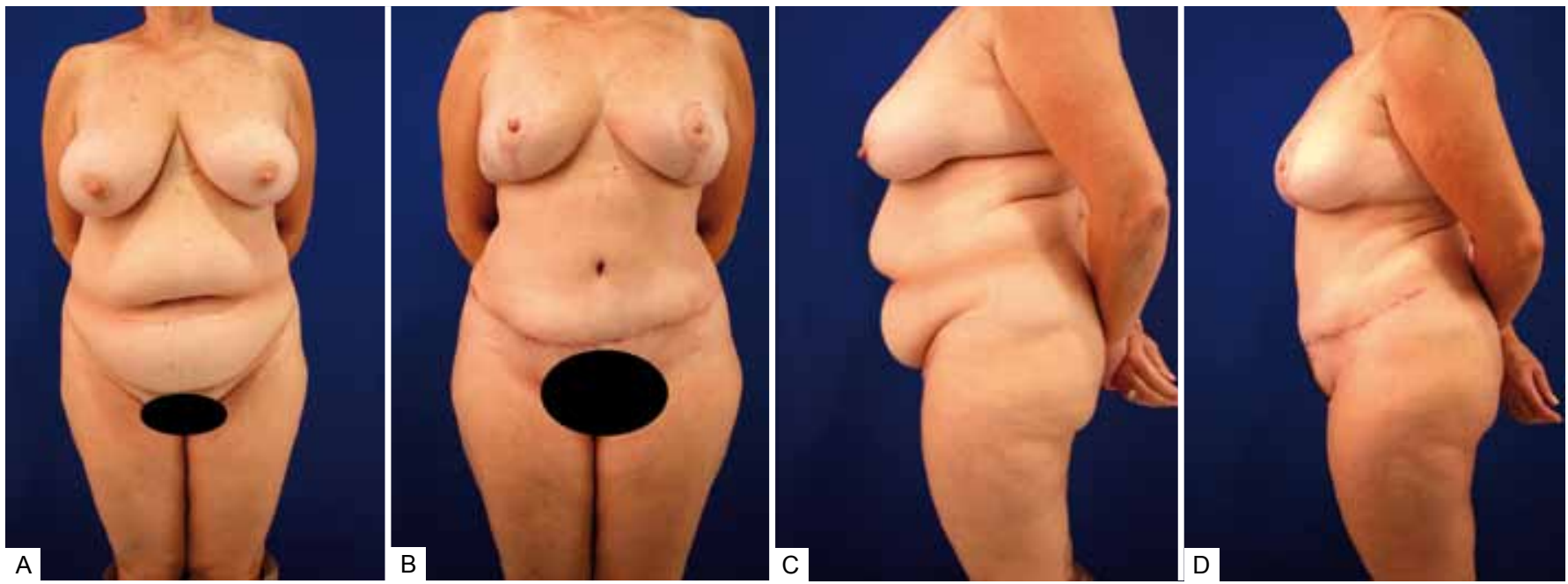

Figura 10 - Lipoabdominoplastia com redução de mamas. A e C: pré-operatório; $\boldsymbol{B}$ e D: pós-operatório.

levantamentos, sendo de $16 \%$ a incidência de TVP e embolia pulmonar, neste último estudo ${ }^{21}$.

Em 2008, Heller et al..$^{22}$ concluíram que a lipoabdominoplastia, com dissecção supraumbilical mediana limitada, produziu menor número de complicações do que a abdominoplastia tradicional. Atribuíram ao fato de reduzir a tensão na linha média de sutura e grande preservação do fluxo vascular para o retalho vindo das artérias intercostais.

Também em 2008, Bins Ely et al. ${ }^{23}$ compararam 10 casos de abdominoplastia e 10 de lipoabdominoplastia, sendo observada a presença de seromas em dois pacientes de cada grupo e um caso de necrose no grupo de abdominoplastia.

Samra et al. ${ }^{24}$, em 2010, observaram taxa de complicações relacionada a perfusão nas lipoabdominoplastias de 4,3\% e, nas abdominoplastias, de 11,76\%. Relataram não haver diferença estatística desses dados entre os pacientes de maior risco, tabagistas ou com cirurgias abdominais anteriores com cicatrizes supraumbilicais. Concluíram que a lipoabdominoplastia não está relacionada a um aumento estatístico nas taxas de complicações relacionadas à perfusão.

Em nossa casuística observamos 20 casos de seroma, 15 casos de cicatrizes inestéticas, com necessidade de revisão cirúrgica, e um caso de infecção de parede abdominal. Não houve hematomas, deiscências, parestesias persistentes e embolia pulmonar.

A colocação de dreno a vácuo foi a única medida per operatória para contenção do seroma. Apesar da complicação mais frequente ter sido o seroma, este foi resolvido com punções aspirativas e compressão, sem trazer queixas.

A utilização regular de heparina de baixo peso molecular foi indicada a pacientes em grupos de risco, tabagistas, idade acima de 60 anos, diabéticas, portadores de doença vascular de membros inferiores, de forma profilática e sendo suspenso ao deambular. Não foi verificado caso de trombose venosa profunda.
A duração do procedimento de lipoabdominoplastia foi de duração relativamente rápida, permitindo a associação de outros procedimentos, de forma segura. O procedimento também foi facilitado pela utilização do vibrolipoaspirador. O resultado estético do contorno corporal ficou mais definido. Para não haver tensão exagerada na porção medial da linha de sutura, algumas vezes, as incisões foram feitas um pouco mais altas do que na abdominoplastia tradicional. Isso levou, nestes casos, à revisão cirúrgica. Entretanto, pelo fato dos pacientes terem sido informados exaustivamente no pré-operatório, não houve relato de insatisfação por parte dos mesmos.

\section{CONCLUSÕES}

O que diferencia este descolamento do realizado nas técnicas descritas de lipoabdominoplastia foi o fato de ser menos conservador no descolamento do retalho supraumbilical e na região periumbilical, atingindo no mínimo $12 \mathrm{~cm}$ de largura, a não preservação da fáscia de Scarpa e a ausência de lipoaspiração do retalho infraumbilical.

Não foi evidenciado nenhum tipo de complicação local intensa, como necrose cutânea ou deiscência. Também não foram observadas complicações sistêmicas, como embolia, atelectasia, infarto ou morte.

$\mathrm{O}$ procedimento de lipoabdominoplastia, envolvendo dorso, flancos e abdome, mostrou ser rápido (3 horas), seguro e de resultado estético satisfatório. A duração do procedimento de lipoabdominoplastia foi alcançada também pela utilização do vibrolipoaspirador.

O resultado estético do contorno corporal ficou mais definido. Para não haver tensão exagerada na porção medial da linha de sutura, as incisões, algumas vezes, foram feitas um pouco mais altas do que numa abdominoplastia tradicional. Isso levou, nestes casos, à revisão cirúrgica. 
Mesmo tendo sido feitos procedimentos associados que acabaram tendo uma duração maior, a lipoabdominoplastia alcançou bons resultados estéticos, satisfação ampla do paciente.

Considero alguns fatores como importantes na preservação vascular do retalho abdominal:

1. O descolamento mais lateralizado superiormente, mesmo comprometendo as perfurantes periumbilicais, é mantido por subcostais, intercostais e perfurantes superiores da artéria epigástrica superior. Desde que não se atinja o rebordo subcostal e a borda lateral dos músculos reto abdominais, a segurança vascular do retalho abdominal descolado e lipoaspirado é preservada;

2. Também a cicatriz horizontal mais alta, sem tensão no fechamento, na porção central, é um fator que evita possíveis necroses cutâneas;

3. Talvez, a utilização do vibrolipoaspirador com cânulas mais finas também tenha facilitado a remoção da gordura sem muito trauma local, preservando o fluxo vascular do retalho.

O fato da ampliação do descolamento ter sido maior do que preconizado e não ter trazido complicações vasculares foi possível com algumas medidas de segurança e também pela anatomia vascular da parede abdominal lateral que se confirmou nas reconstruções mamárias.

\section{REFERÊNCIAS}

1. Pitanguy I. Abdominal lipectomy: an approach to it through an analysis of 300 consecutives cases. Plast Reconstr Surg. 1967;40(4):384-91.

2. Callia WEP. Contribuição para o estudo da correção cirúrgica do abdômen pêndulo e globoso: técnica original [Tese de Doutoramento]. São Paulo: Faculdade de Medicina da Universidade de São Paulo;1965.

3. Illouz YG. A new technique for the localized lipodistrophies. Es Ver Chir Esth France 6,1980 .

4. Mathes SJ, Nahai F. Clinical applications for muscle and musculocutaneous flaps. St Louis;Mosby;1982. 733p.

5. Huger WE Jr. The anatomic rationale for abdominal lipectomy. Ann Surg. 1979;45(9):612-7.

6. Hakme F. Technical details in the lipoaspiration associate with liposuction. Rev Bras Cir. 1985;75(5):331-7.

7. Shestak KC. Marriage abdominoplasty extands the mini-abdominoplasty concept. Plast Reconstr Surg. 1999;103(3):1020-31.
8. Avelar JM. Abdominoplasty: a new technique without undermining and fat layer removal. ACM Arq Catarin Med. 2000;29:147-9.

9. Saldanha OR, Pinto EB, Mattos WN Jr, Lucon RL, Magalhães F, Bello EM. Lipoabdominoplasty without undermining. Aesthet Surg J. 2001;21(6):518-26.

10. Saldanha OR, De Souza Pinto EB, Mattos WN Jr, PazettiCE, Lopes Bello EM, Rojas Y. Lipoabdominoplasty with selective and safe undermining. Aesthetic Plast Surg. 2003;27(4):322-7.

11. Graf R, de Araujo LR, Rippel R, Neto LG, Pace DT, Cruz GA. Lipoabdominoplasty: liposuction with reduced undermining and traditional abdominal skin flap. Aesthetic Plast Surg. 2006;30(1):1-8.

12. Uebel CO. Lipoabdominoplasty: revisiting the superior pulldown abdominal flap and new approaches. Aesthetic Plast Surg. 2009;33(3):366-76.

13. Vila-Rovira R. Lipoabdominoplasty. Clin Plast Surg. 2008;35(1):95104.

14. El-Mrakby HH, Milner RH. The vascular anatomy of the lower anterior abdominal wall: a microdissection study on the deep inferior epigastric vessels and perfurators branches. Plast Reconstr Surg. 2002;109(2):539-43.

15. Boyd JB, Taylor GI, Corlett R. The vascular territories of the superior epigastric and deep inferior epigastric systems. Plast Reconstr Surg. 1984;73(1):1-16.

16. Taylor GI, Watterson PA, Zelt RG. The vascular anatomy of the anterior abdominal wall: the basis for flap design. Perspect Plast Surg. 1991;5:1-30,

17. Matarasso A, Swift RW, Rankin M. Abdominoplasty and abdominal contour surgery: a national plastic surgery survey. Plast Reconstr Surg. 2006;117(6):1797-808

18. Alderman AK, Collins ED, Streu R, Grotting JC, Sulkin AL, Neligan P, et al. Benchmarking outcomes in plastic surgery: national complication rates for abdominoplasty and breast augmentation. Plast Reconstr Surg. 2009;124(6):2127-33.

19. Stevens WG, Spring MA, Stoker DA, Cohen R, Vath SD, Hirsch EM. Ten years of outpatient abdominoplasties: safe and effective. Aesthet Surg J. 2007;27(3):269-75.

20. Stewart KJ, Stewart DA, Coghlan B, Harrison DH, Jones BM, Waterhouse N. Complications of 278 consecutive abdominoplasties. J Plast Reconstr Aesthet Surg. 2006;59(11):1152-5.

21. Neaman KC, Hausen JE. Analysis of complications from abdominoplasty: a review of 206 cases at a university hospital. Ann Plast Surg. 2007;58(3):292-8.

22. Heller JB, Teng E, Knoll BI, Persing J. Outcome analysis of combined lipoabdominoplasty versus conventional abdominoplasty. Plast Reconstr Surg. 2008;121(5):1821-9.

23. Bins-Ely J, Duarte FO, Vieira VJ, Vasconcellos ZAA, Neves RD. Comparação entre abdominoplastia convencional e lipoabdominoplastia: abdominoplastia versus lipoabdominoplasty. ACM Arq Catarin Med. 2008;37(4):40-5.

24. Samra S, Sawh-Martinez R, Barry O, Persing JA. Complication rates of lipoabdominoplasty versus tradicional abdominoplasty in high-risk patients. Plast Reconstr Surg. 2010;125(2):683-90. 